\title{
Use of Video for Teaching Sports Mechanics ${ }^{\dagger}$
}

\author{
John Eric Goff 1,* and Tom Allen ${ }^{2}$ \\ 1 Department of Physics, University of Lynchburg, Lynchburg, VA 24501, USA \\ 2 Department of Engineering, Manchester Metropolitan University, Manchester M15 6BH, UK; \\ t.allen@mmu.ac.uk \\ * Correspondence: goff@lynchburg.edu; Tel.: +1-434-544-8856 \\ + Presented at the 13th conference of the International Sports Engineering Association, Online, \\ 22-26 June 2020.
}

Published: 15 June 2020

\begin{abstract}
There is a shortage of young people studying physics and engineering and these professions could benefit from greater diversity. Sports engineering is often used as a tool to change the public perceptions of engineering and sporting examples can be used in physics and engineering classes to engage people. Video analysis is widely used by sports engineers, and this publication showcases how the video analysis of sporting scenarios can be used to teach mechanics. Examples illustrate how the movement of an athlete and equipment can be analysed and linked to basic physics, for sports ranging from sumo to soccer. The examples presented can be used for teaching and outreach, with a view to enthuse students about physics and engineering.
\end{abstract}

Keywords: education resources; physics of sport; sports engineering

\section{Introduction}

A core competency shared by engineering and physics is the ability to model the natural world. Modelling the sports world is this paper's focus. It would be hard to engage students in a sports engineering or sports physics course if they were not exposed to real sports examples. Previous papers provided an overview of resources with sports examples to support engineering [1] and physics [2,3] teaching. Using photos [4] and videos is a pedagogical must. The ease of accessing free online sports clips allows educators to infuse their courses with videos from a plethora of sports, to match the needs of class topics and student interests. Courses will not become dated because new spor videos are available daily, allowing educators to design courses with videos that showcase current sports stars as well as the latest technologies. Educators may also highlight amazing plays that could only be a day or two old when they are discussed in class.

Though not limited to one scientific field, this paper restricts its discussions to using video analysis in the field of physics known as "classical mechanics," or "mechanics" for short. This paper also follows the spirit of previous pedagogical papers [4-6] presented at ISEA conferences by describing teaching ideas that were supported by anecdotal evidence. Sport videos may not only serve as a tool to generate interest and discussion around the physics of sport, they may also be used for a basic analysis of the mechanics of a given move or play. Video analysis is used by scientists and engineers across numerous sports [7-10]. Though many researchers and practitioners now use automated video analysis, manual analysis is well suited to teaching. The focus of teaching is to ensure that students develop a fundamental understanding, which means only a few scenarios need to be analysed. Freely available tracking software, such as Tracker [11], is ideal for teaching as it allows unrestricted access. Using such software to track videos, students can familiarise themselves with concepts such as two-dimensional (2D) calibration and the measurement of object size and displacement, as well as the conversion of displacement to velocity using the known frame rate and 
the estimation of error. This paper uses examples from sports ranging from sumo to soccer to showcase how videos can support the teaching of sports mechanics.

\section{Teaching the Basics}

Instructors should take at least an hour to acclimate students to the use of video and how to analyse it. Modern phones record a video quality of 240 frames per second (fps), which means a recording action in $4.2 \mathrm{~ms}$ time intervals. Even older phones and more traditional cameras that record at $30 \mathrm{fps}$, which translates to frames taken every $33 \mathrm{~ms}$, are sufficient for getting students started on video analysis. Cameras typically allow users to record motion in one plane. Students thus need to shoot the video while at a reasonable distance from the action, to prevent out-of-plane errors and so that the video is not recorded with light coming into the camera at large angles. The camera must remain stationary; otherwise, calibration will be lost. Students should be warned when obtaining videos from online that they will only be able to analyse parts of the video for which the camera is relatively still, and that no zooming in or out takes place. A reference length needs to be placed in the plane of motion, so that the video software can translate the motion across pixels into actual distances. Students may not appreciate how much effort goes into preparing to record video quality suitable for analysis. These efforts are usually made in tandem with someone else who has agreed to be filmed.

The easiest type of motion one may imagine is tossing or dropping an object, be it a ball or something else. Before students learn about the complexities of air resistance, they learn the basics of projectile motion in a vacuum. They also employ the so-called "spherical-cow approximation" in which everything they model is a point particle. They need to understand how Newton's laws work for point particles before tackling inertia tensors and rigid-body motion. A good first example is that of a tossed baseball bat (or a similar, extended object). Figure 1a shows one frame from the video of a tossed baseball bat, filmed at $60 \mathrm{fps}$. The centre of mass was clearly marked on the bat, so that its position could be tracked for each frame of the motion after it was tossed and before it hit the ground. Before the toss, the bat was recorded lying on the ground in the plane of its soon-to-be tossed motion, so a $2 \mathrm{D}$ calibration could be performed. Its $81 \mathrm{~cm}$ length is marked in blue in Tracker (bat was on the ground earlier in the video). Cartesian coordinate axes are in purple with the origin in the lower left portion of the image. After the bat's centre of mass was marked for each frame, Tracker's complete data table and finished graphs could be viewed. Modelling the motion of the bat's centre of mass to that of a point particle undergoing projectile motion under the sole influence of a constant gravitational field led to the blue curve in Figure 1b's graph (actual data are shown as red dots). The quadratic modelling function led to a magnitude of the acceleration due to gravity, $g$, of $9.838 \mathrm{~m} / \mathrm{s}^{2}$, just $0.4 \%$ off from the actual value.

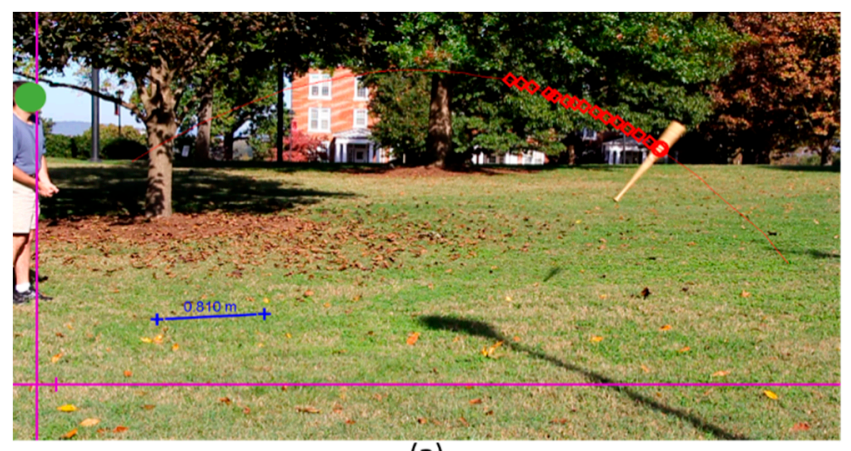

(a)

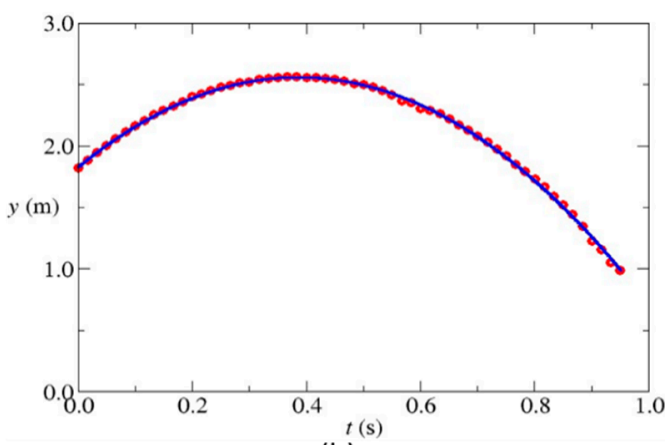

(b)

Figure 1. (a) Baseball bat tossed with rotation; (b) the vertical position of the bat's centre of mass vs. time.

Many things may be gleaned from the baseball bat toss example. One is a demonstration of how the centre-of-mass motion of the bat gets separated from the more complicated rotating motion around its centre of mass. Because Tracker allows the user to keep the tracked path on the screen, 
students may view the parabola they derived using vacuum kinematics. Despite the relatively small error in $g$, students should realise that there are left-right-up-down errors in marking a given point in a given frame. These errors can be estimated by tracking the video a few times, or by comparing tracking between students. These errors do, however, tend to average out when the data are fitted to a model function. Students should also be made aware of the mathematical uncertainties intrinsic to a model's fitting function. Even uncertainty in a measured quantity to be modelled with least-squares linear fitting leads to uncertainties in the fitting coefficients [12]. Students may modify the length of the reference length and watch how the data and the fitting equation change. They may also study the motion of an object frame by frame to get a better understanding of what takes place during the complicated motion. This might not be so interesting with the tossed-bat example, but watching a gymnast or snowboarder [13] execute various movements frame by frame may fascinate students. Beyond what the science educators are trying to teach, athletes taking a sports engineering or sports physics course may be enlightened by a new way to enhance their performances.

Educators need not put a simple example like that of a tossed bat away after one class. One obvious extension is to have students return to the video when learning about rigid-body motion. By tracking multiple points, students could then study the motion of the bat around its centre of mass. Even before moving away from single-particle physics, instructors could have students determine the translational kinetic energy of the bat's centre of mass. Such a calculation is easy in Tracker because the software allows the user to define the parameters and create equations that may be plotted. Once the graphs of the user's choosing are displayed next to the tracked image, one may step frame by frame, watching the video and marker on each point on the graph advance. This feature allows an instructor to get to an ideal frame in a video, and then discuss what is happening while pointing to various values in accompanying graphs, which have markers on data indicating where in the graph the video has been stopped.

\section{Examples}

The following subsections describe examples that have been used in teaching mechanics in sports engineering and sports physics courses. All of the examples assumed that the students have learned the basics of video analysis as described in the previous section.

\subsection{Rugby}

The quickest try from kick-off in international rugby occurred on 2 June 2014 in New Zealand. Welsh winger Dafydd Howells crossed the try line six seconds after kick-off in a match against Fiji. An analysis of the play was published in [14]; a brief summary of that analysis will be given here. Tracker was used with a video of the play to determine the launch speed and angle of the rugby ball upon kick-off. By analysing the bounce of the ball off the pitch, the coefficient of restitution (CoR) was determined and found to be consistent with values published for the rugby balls.

Students may be introduced to CoR with video analysis. Simply film a ball dropped onto the floor, similar to Figure 1 in [4] (an open-access reference) as captured with a stroboscopic technique. $\mathrm{CoR}$ is the absolute value of the ratio of the relative velocity of two objects after they collide to their relative velocity prior to collision. For a solid floor, CoR is simply the square root of the ratio of the ball's post-bounce height to its drop height. Moving frame by frame in Tracker makes it easy to find a ball's maximum post-bounce height. However, once students are introduced to CoR, they should be told that that quantity, like friction, is tied to the two objects undergoing the collision. One could find the CoR between a rugby ball and the solid floor, but that number would only be of pedagogical interest. Bouncing the rugby ball off a rugby pitch would lead one to determine a CoR that would be of interest to anyone associated with the science of rugby. That is why students should be introduced to the type of video analysis associated with real sporting action, as described at the beginning of this subsection. 


\subsection{Softball}

Pitching, be it in baseball or softball, is challenging to analyse because of the complexities associated with the pitcher's movements. A pitcher's entire body is involved in throwing the ball. Though those bodily motions are not confined to a plane, much may be gleaned from a video taken from the side. Figure 2 shows a frame from video of a pitcher $0.13 \mathrm{~s}$ before releasing the ball.

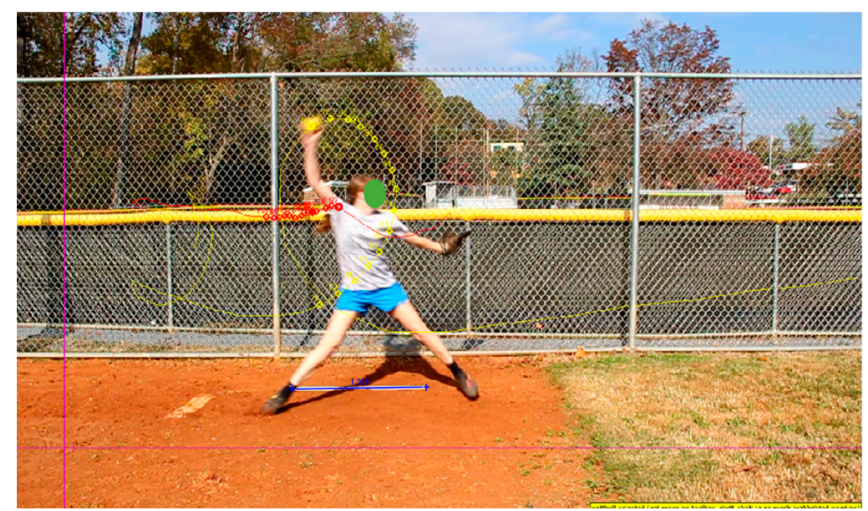

Figure 2. Softball player in the act of pitching. Both the softball and the pitcher's right shoulder were tracked.

The large rotational arcs of the ball in hand could easily be tracked. By also tracking the pitcher's right shoulder, students could analyse how the forward motion of the pitcher's body played a role in the ball's launch speed. Using basic physics they had already been taught [15], the students found that the pitcher's shoulder reached a speed of nearly $5 \mathrm{~m} / \mathrm{s}$ just prior to the ball's release. The ball left her hand at approximately $28 \mathrm{~m} / \mathrm{s}$ with a translational kinetic energy of almost $75 \mathrm{~J}$. By analysing the ball's acceleration during the pitching motion, students were able to find a peak acceleration magnitude of nearly $47 \times \mathrm{g}$. Based on the ball's mass of $0.188 \mathrm{~kg}$, it was deduced that the pitcher's right arm had to exert a force of over $88 \mathrm{~N}$ at the bottom of the swing to maintain an acceleration of $47 \times \mathrm{g}$. The pitcher's arm's rotational speed hit $30 \mathrm{rad} / \mathrm{s}$, which is about two-thirds of the rotational speed of helicopter blades. Such comparisons are helpful in getting students familiar with the orders of magnitude of a myriad of physical quantities, such as force, length, and speed.

\subsection{Sumo}

The availability of videos from around the world makes it possible to bring unfamiliar sporting scenarios to the classroom. Instructors teaching outside of Japan may not be familiar with sumo, which has a professional history more than four centuries old. Students may analyse sumo video for many physic aspects of the sport. Mitakeumi Hisashi, with a mass of $177 \mathrm{~kg}$, won the September 2019 top-division championship over Takakeishō Mitsunobu, who had a mass of $169 \mathrm{~kg}$. When the two rikishi collided in the final match, it appeared as if there was no net linear momentum in the system. However, Mitakeumi maintained his backward push against the ground, which enabled him to continue driving into Takakeishō. Figure 3a shows the initial collision and the moment just half a second later (Figure 3b) when Mitakeumi had the upper hand.
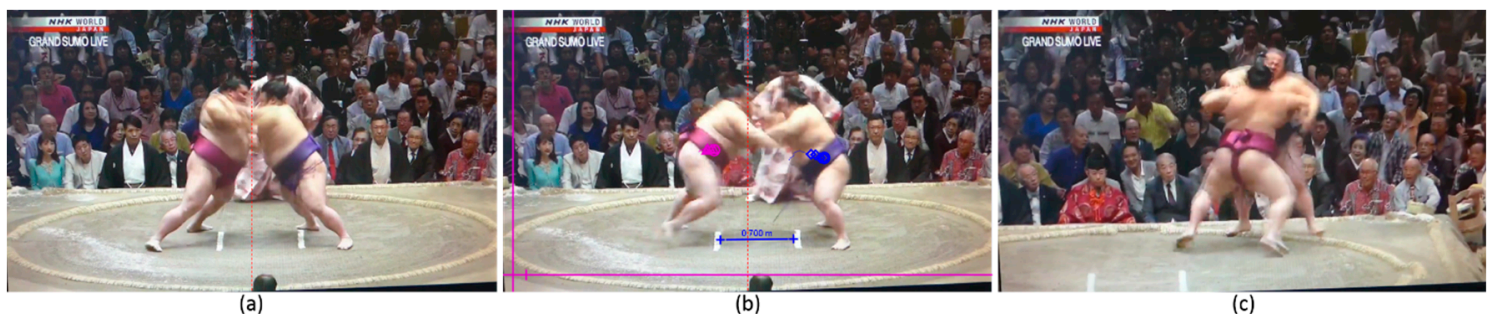

Figure 3. (a) Mitakeumi (left) and Takakeishō (right); (b) Mitakeumi had linear momentum to the right, which he needed to get the upper hand on Takakeishō; (c) Mitakeumi winning. 
Tracker was used to compute the sum of the linear momenta of the two rikishi at the moment of initial impact (Figure 3a). It was essentially zero at that point, but when the combatants separated slightly, Mitakeumi got a great push off the floor that allowed him to drive forward. This is an excellent teaching moment. Students sometimes hear phrases like "conservation of linear momentum" and think that linear momentum is conserved all the time. However, of course the net linear momentum in a system is conserved only if there is no net force on the system. If the two rikishi were considered to be the "system" under consideration, it appeared as if their respective friction forces cancelled at the time of the initial collision. So, when students used basic physics [15] to calculate the initial linear momentum of each athlete, they found that the system linear momentum was indeed zero. However, that was not the case with the second image (Figure 3b). The floor's friction force on Mitakeumi was larger than the friction force on his opponent. That meant that the system comprising of the two rikishi had a net force on it, which is why the system moved to the right in Figure 3b. Note that a previous comment in this paper about athletes using video and their knowledge of physics to improve performances could certainly be applied to the match shown in Figure 3.

Further analysis on Tracker showed how Mitakeumi was able to push Takakeishō out of the ring. Mitakeumi lowered his centre of mass and pushed upward at just the right moment to lift Takakeishō for the winning push. The winning moment happened at the back of the ring (Figure 3c), making the analysis of speeds nearly impossible, but it was easy to dissect the physics involved in the winning move. The use of Tracker is not always confined to extracting numbers.

\subsection{Soccer}

Analysing soccer is challenging because action rarely happens in one plane. Player movements are complicated and balls kicked in the air usually have spin and thus do not have 2D trajectories. Most online soccer videos were not filmed with video analysis in mind. It may thus take an instructor a little time to find a soccer video suitable for analysis. One such video was found during the 2014 World Cup in Brazil. Near the end of the first half in a match against Nigeria, Lionel Messi executed a nearly flawless free kick. The video from two vantage points allowed for the analysis of Messi's kick to be performed. Figure 4 shows a frame from a video shot at an elevated view and a frame from a video taken at pitch level.

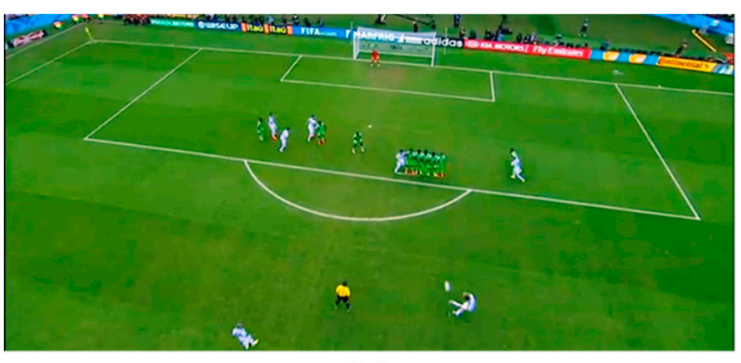

(a)

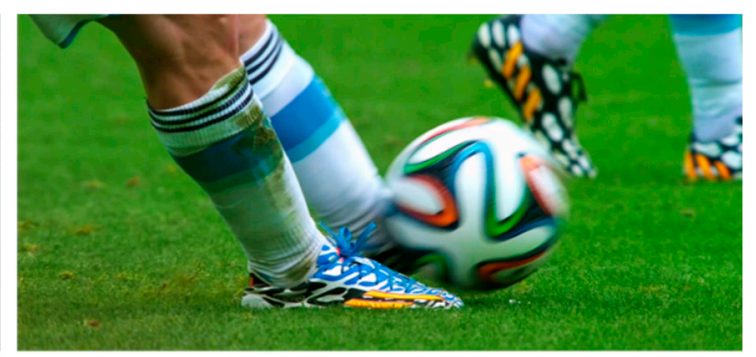

(b)

Figure 4. A Lionel Messi free kick during the 2014 World Cup; (a) the top view; (b) the pitch-level view.

The ball left Messi's boot and then curled into the right side of the goal $1.2 \mathrm{~s}$ later. The analysis of the videos from which the images in Figure 4 were derived revealed that the ball left Messi's boot $18.5^{\circ}$ above the horizontal at a speed of $24.8 \mathrm{~m} / \mathrm{s}$. As would have been seen from above, the ball contained a clockwise spin, creating Magnus force on the ball during its flight. The analysis of Messi's kick in the classroom focused first on the kick itself. His left boot drove through the ball left of centre, which created the necessary torque on the ball to get it spinning. The angle of his planted right leg led to a discussion on stability while kicking. The pitch provided the counter-torque that kept Messi stable because his right boot pushed to his left, which meant the pitch pushed his boot to his right. That counter-torque from the pitch nearly balanced the torque from his weight, with his arm movements providing additional stabilization. 
Once the kick itself was analysed, the students were then asked to use the initial position and initial velocity to model the flight of the ball. Though Tracker could only help with a mostly qualitative overhead view of the kick, more traditional computational techniques were employed to account for air resistance and the Magnus force. It was found that had the ball not been spinning, it would have crossed the goal plane $3.28 \mathrm{~m}$ left of where it actually crossed the goal plane. Air resistance reduced the ball's speed to $18.8 \mathrm{~m} / \mathrm{s}$ as it crossed the goal plane.

\section{Summary}

This paper served to contribute to previous ISEA conference submissions that fall under the category of "Education of Sports Engineering." By employing freely available software, such as Tracker, educators will be able to have students analysing sport videos after only a relatively quick class session to introduce the software. Students will then use their basic physics knowledge to extract a myriad of useful information from sport videos. The fact that numerous contemporary sport videos are uploaded every day allows instructors to keep their courses fresh and congruent with student interests. Examples of how Tracker may be used to tease out both the qualitative and the quantitative facts from videos were presented in this work. The six-page paper limit prevented more detailed descriptions of how to utilize Tracker, but the software is quite easy to acquire and use. Extensions of the work presented here will involve monitoring this paper's prescribed pedagogical methods and their impact on several renditions of courses in sports engineering and sports physics. Acquiring student data will be part of such a future study, which will be published in a more traditional journal, one that does not have the constraints set for papers aimed at an audience of conference participants.

Funding: No funding was received for this work.

Conflicts of Interest: The authors declare no conflict of interest.

\section{References}

1. Allen, T.; Goff, J.E. Resources for sports engineering education. Sports Eng. 2018, 21, 245-253.

2. Frohlich, C. Resource letter PS-1: Physics of sports. Am. J. Phys. 1986, 54, 590-593.

3. Frohlich, C. Resource letter PS-2: Physics of sports. Am. J. Phys. 2011, 79, 565-574.

4. Driscoll, H.; Hart, J.; Allen, T. Use of image based sports case studies for teaching mechanics. Proc. Eng. 2016, 147, 884-889.

5. Medwell, P.R.; Grimshaw, P.N.; Robertson, W.S.; Kelso, R.M. Developing sports engineering education in Australia. Proc. Eng. 2012, 43, 260-265.

6. Petrone, N.; Marcolin, G.; Odenwald, S.; Senner, V. The ISEA Winterschool in Sports Engineering: 5 years of education and team building experiences. Proc. Eng. 2016, 147, 896-900.

7. Barris, S.; Button, C. A review of vision-based motion analysis in sport. Sports Med. 2008, 38, 1025-1043.

8. Elliott, N.; Choppin, S.; Goodwill, S.; Senior, T.; Hart, J.; Allen, T. Single view silhouette fitting techniques for estimating tennis racket position. Sports Eng. 2018, 21, 137-147.

9. Dunn, M.; Kelley, J. Non-invasive, spatio-temporal gait analysis for sprint running using a single camera. Proc. Eng. 2015, 112, 528-533.

10. Goff, J.E.; Kelley, J.; Hobson, C.H.; Seo, K.; Asai, T.; Choppin, S.B. Creating drag and lift curves from soccer trajectories. Eur. J. Phys. 2017, 38, 044003.

11. Tracker Video Analysis and Modeling Tool. Available online: https://physlets.org/tracker/ (accessed on 19 September 2019).

12. Taylor, J.R. An Introduction to Error Analysis: The Study of Uncertainties in Physical Measurements, 2nd ed.; University Science Books: Sausalito, CA, USA, 1997; pp. 186-190.

13. Chen, S. The physics of flip and spin. WIRED 2018, 26, 20-21. 
14. Goff, J.E.; Lipscombe, T. Trying physics: Analyzing the motion of the quickest score in international rugby. Phys. Teach. 2015, 53, 72-74.

15. Wolfson, R. Essential University Physics, 4th ed.; Pearson: Hoboken, NJ, USA, 2020.

(c) (P) BY
(C) 2020 by the authors. Licensee MDPI, Basel, Switzerland. This article is an open access article distributed under the terms and conditions of the Creative Commons Attribution (CC BY) license (http://creativecommons.org/licenses/by/4.0/). 